\title{
ВОЗМОЖНОСТИ ПОВЫШЕНИЯ УРОВНЯ ТЕХНОЛОГИЧЕСКОЙ ЭФФЕКТИВНОСТИ ДЕЙСТВУЮЩИХ ТЕХНИЧЕСКИХ ОБЪЕКТОВ
}

\author{
Воинова C. A. ${ }^{1}$ \\ ${ }^{1}$ Одесская национальная академия пищевых технологий, Одесса \\ Copyright ( $\odot 2014$ by author and the journal "Automation technological and business - processes". \\ This work is licensed under the Creative Commons Attribution International License (CC BY). \\ http://creativecommons.org/licenses/by/4.0/
}

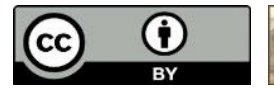

\begin{abstract}
Аннотация
В отечественном производстве значительная часть действующего технологического оборудования израсходовала расчетный ресурс, поэтому функционирует с невысокими технологическими показателями. Их повышение возможно путем частичного обновления оборудования. Реализация подобной задачи отличается значительной рентабельностью при относительно невысокой ресурсоемкости. Программа работ по обновлению нуждается в разработке и применении средств оперативного контроля составляющих технологической эффективности обновляемых технических объектов и в оценке возможной эффективности их применения. Реализация подобной программы нуждается в усилении социальной ответственности технического персонала всех уровней, ответственного за качество процесса функционирования изношенного оборудования.
\end{abstract}

\section{Abstract}

Abstract: In a home production the considerable part of operating processing equipment spent a settlement resource therefore it functions with low technological indicators. Their increase is possible by partial updating of the equipment. Realization of a similar task differs considerable profitability at rather low resource intensity. The program of works for updating needs development and application of means of an operating control of updated technical objects making to technological efficiency and an assessment of possible efficiency of their application. Implementation of the similar program needs strengthening of social responsibility of technicians of all levels responsible for quality of process of functioning of the worn-out equipment.

\section{Ключевые слова}

Технический объект, повышение, технологическая эффективность, управление, процесс, расходование, pecypc.

\section{Состояние вопроса}

Продолжающийся стагнационный период развития мирового производства повысил до высокого уровня долю производственных фондов, израсходовавших ресурс работоспособности, поскольку действующие технические объекты (ТО), от момента ввода в действие, подвержены воздействию процесса износа. Вследствие этого, происходит деградация технологических свойств ТО, соответственно этому снижается уровень его технологической эффективности $\left(\mathrm{E}_{\mathrm{T}}\right)$ от уровня исходного (максимального) ( $\mathrm{E}_{\text {исх }}$ ) до уровня минимально допустимого $\left(\mathrm{E}_{\text {мин }}\right)$. Этот процесс занимает период времени, равный продолжительности ресурса работоспособности ТО, назначенного его заводом-изготовителем. 


\section{$\underline{1}$ ПИТАННЯ ТЕОРІЇ, МЕТОДИ ТА АЛГОРИТМИ ЕФЕКТИВНОГО АВТОМАТИЧНОГО УПРАВЛІННЯ ОБ’ЄКТАМИ ХІМІКО-ТЕХНОЛОГІЧНОГО ТИПУ}

Прогрессивный подход к управлению процессом функционирования ТО

При прогрессивном подходе к управлению процессом функционирования ТО, формирование $\mathrm{E}_{\mathrm{T}}$ этого процесса осуществляют в три этапа. В их составе - технологический, конструкторский и оперативный этапы. Первый и второй образуют накопительный период, третий - расходный период $[1,2]$.

Условимся, далее понятие « $\mathrm{E}_{\mathrm{T}}$ процесса функционирования ТО» представлять в сокращении «Е

Ет сформирована тремя составляющими: экологической $\mathrm{E}_{л}$, экономической $\mathrm{E}_{\mathrm{H}}$ и общетехнической $\mathrm{E}_{\text {общ: }}$

$\mathrm{E}_{\mathrm{T}}=\mathrm{f}\left(\mathrm{E}_{\mathrm{J}}, \mathrm{E}_{\mathrm{H}}, \mathrm{E}_{\text {общ}}\right)$. В каждом конкретном случае для каждого из этих показателей устанавливают минимально допустимые уровни $\left(\mathrm{E}_{\mathrm{J}}\right)_{\min },\left(\mathrm{E}_{\mathrm{H}}\right)_{\min },\left(\mathrm{E}_{\text {общ }}\right)_{\min }$. Из наборов свойств каждой из них выбирают основное свойство (показатель) $\mathrm{E}_{\mathrm{T}}$ ТО. По этому свойству, в конечном счете, назначают ресурс ТО, по экологическим, по экономическим и по общетехническим свойствам. Для этого, необходимо располагать сведениями о закономерностях изменения во времени выбранных трех показателей эффективности под действием процесса износа ТО. Эти принципиально важные закономерности являются предметом изучения в рамках технической геронтологии [3].

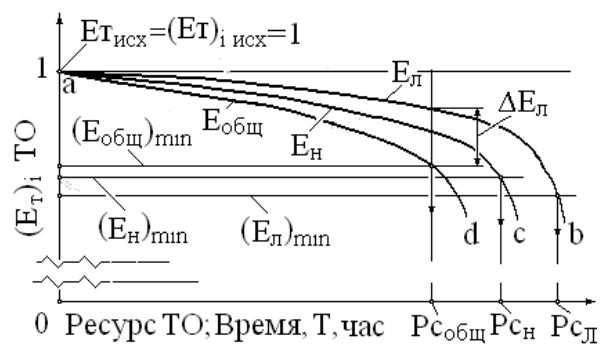

Рис. 1 - Пример соотношения расчетных размеров $\mathrm{Pc}_{Л}, \mathrm{Pc}_{\mathrm{H}}$ и $\mathrm{Pc}_{\text {ОБщ }} \mathrm{TO}$
Графо-аналитический подход к определению ресурса ТО

Опираясь на известные закономерности влияния процесса износа ТО на каждое из выделенных трех основных его свойств, для каждого из них строят траекторию движения ТО в координатах «Е $\mathrm{E}_{\mathrm{T}}-$ время» $\left(« \mathrm{E}_{\mathrm{T}}-\mathrm{T} »\right)$ (рис.). В этих же координатах отмечают минимально допустимый уровень каждого из трех основных свойств ТО $\left(\mathrm{E}_{\text {л }}\right)_{\min }$, $\left(\mathrm{E}_{\mathrm{H}}\right)_{\min },\left(\mathrm{E}_{\text {общ }}\right)_{\min }$.

Далее, на графике $\mathrm{E}_{л}(\mathrm{~T})$ находят точку (момент времени), где $\mathrm{E}_{л}$ снижается до уровня $\left(\mathrm{E}_{л}\right)_{\min }$. Этот момент времени отмечает (отсчитывает) размер ресурса ТО по экологическим свойствам - $\mathrm{Pc}_{л}$.

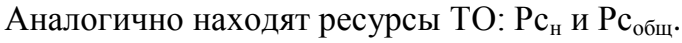

В общем случае размер найденных трех ресурсов различен.

Полученные размеры ресурсов необходимо ранжировать по степени важности в конкретных, заданных условиях применения ТО. Ресурсом ТО логично считать выявленный ресурс по главному из трех исследованных основных его свойств. В примере на рисунке главным является ресурс по экологическим свойствам ТО - $\mathrm{Pc}_{л}$.

Исходя из необходимости исключить возможность использования ТО после исчерпания им ресурса (то есть ресурса по главному свойству), необходимо, чтобы его размер превосходил размеры ресурсов по двум другим, основным свойствам. При этом условии ТО достигнет исчерпания ресурса по одному из двух других свойств и будет выведен из эксплуатации. При этом, у него фактически еще будет неиспользованным остаточный ресурс по главному свойству. То есть, не будет допущено снижение текущего уровня главного технологического свойства ниже его принятого минимального допустимого уровня по главному свойству. Это - результат принципиально важный.

В качестве примера, приведен случай, когда ресурсом ТО был определен ресурс по экологическому свойству - $\mathrm{Pc}_{л}$ (Рис.). Данный объект необходимо вывести из эксплуатации по исчерпании им ресурса Р $\mathrm{c}_{\text {общ }}$ (например, по надежности). У этого ТО в момент вывода его из эксплуатации остаточный ресурс составит период (Рс $\left.\mathrm{c}_{л}-\mathrm{Pc}_{\text {общ }}\right)$.

Влияние режима работы ТО на определение ресурса

При регулярном режиме работы ТО, среднестатистически, изменение его $\mathrm{E}_{\mathrm{T}}$ будет соответствовать найденным траекториям $\mathrm{E}_{\mathrm{T}}(\mathrm{T})$, как показано выше.

В условиях реального производства характерен нерегулярный режим, когда происходят случайные отклонения от регламентных условий работы: нарушения режима и условий функционирования ТО, отказы, аварии и др. Подобные внерегламентные нарушения негативно влияют на состояние ТО, на его свойства и, как следствие, снижают (скачкообразно, ступенчато) эффективность процесса его функционирования. Причем, размер ущерба, наносимого указанными внерегламентными воздействиями, каждой из составляющих - $\mathrm{E}_{л}, \mathrm{E}_{\mathrm{H}}$ и $\mathrm{E}_{\text {общ, }}$ различен. В подобных условиях уровень $\mathrm{E}_{\mathrm{T}}$ производства низок, особенно если оборудование завершило расходование ресурса, то есть, перешло в предельное состояние. В значительной части отраслей отечественного производства, в том числе в энергетике, подобное оборудование составляет основную часть парка ТО. 


\section{1 ПИТАННЯ ТЕОРІЇ, МЕТОДИ ТА АЛГОРИТМИ ЕФЕКТИВНОГО АВТОМАТИЧНОГО УПРАВЛІННЯ ОБ’ЄКТАМИ ХІМІКО-ТЕХНОЛОГІЧНОГО ТИПУ}

Проблема повышения эффективности функционирования действующего оборудования

Накопленный опыт свидетельствует, что действующие ТО располагают определенным резервом повышения уровня их $\mathrm{E}_{\mathrm{T}}$. Использовать эту возможность тем более целесообразно, чем ниже уровень эффективности ТО, то есть чем более они изношены.

В сложившихся сложных условиях, возможность использования резерва повышения эффективности процесса функционирования действующего изношенного оборудования приобрела острую актуальность. Сформировалась сложная и важная проблема повышения эффективности функционирования действующих ТО, управлением их состоянием и режимом функционирования. Для решения проблемы, необходимо в пределах видимой перспективы, в период замены изношенного оборудования новым высокоэффективным инновационно насыщенным оборудованием, улучшить состояние действующего изношенного оборудования, изыскать и использовать резервы повышения его технологической эффективности.

Необходимо обеспечить осуществление программы мероприятий, направленных на изучение изношенных ТО, контроль текущего уровня их $\mathrm{E}_{\mathrm{T}}$, ее составляющих, изыскание и использование резерва, реальных возможностей повышения показателей функционирования этих объектов. Фактически необходимо и крайне целесообразно осуществить частичное обновление изношенного оборудования, нормализовать режим его эксплуатации, повысить качество технического обслуживания, повысить качество процесса управления технологическим процессом, осуществляемого системами автоматического управления [4].

Подобные возможности реально существуют. Они доступны и относительно мало ресурсоемки. Для их использования, необходимо проводить комплекс мероприятий, вполне доступных в отношении финансовых затрат, но требующих усиления социальной, ответственности работников, психологической и организационнотехнической обстановки на производственном участке, повышения уровня технологической культуры во всех ее проявлениях.

Задача оперативного определения текущего состояния действующего ТО

В проблеме повышения эффективности действующего изношенного оборудования важное значение имеет задача оперативного определения текущего состояния конкретного действующего ТО, то есть оперативного измерения уровня его $\mathrm{E}_{\mathrm{T}}$. Для решения этой задачи, необходимо оперативно измерить уровень составляющих $\mathrm{E}_{\mathrm{T}}$, а

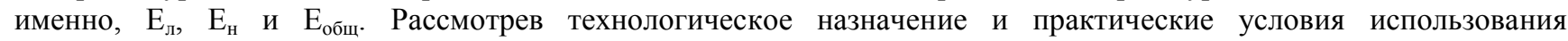
исследуемого ТО, с позиций действующих нормативных положений, следует дать критическую оценку

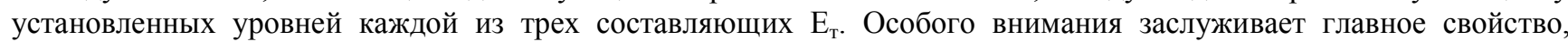
определяющее уровень $\mathrm{E}_{\mathrm{T}} \mathrm{TO}$,- его остаточный ресурс.

Далее, необходимо критически оценить текущее состояние ТО, наметить комплекс технических мероприятий, которые позволят обновить ТО, использовать наличный резерв доступного повышения уровня его Е достойное место должно быть отведено сфере управления производственным участком и расположенным в нем обновляемым ТО.

Так можно представить алгоритм управления программой повышения эффективности изношенного оборудования.

Особенности программы обновления действующих ТО

Важно обеспечить такое положение, при котором коррективы, вносимые в регламент эксплуатации и технического обслуживания ТО, а также в постановку работы обслуживающего ТО персонала, были бы доступными, посильными для него и для предприятия. Подобное решение задач рассматриваемой проблематики позволит наиболее целесообразно использовать ТО в период до предстоящего его списания.

Следует особо отметить то важное обстоятельство, что разумно разработанное (по содержанию и по объему) обновление действующего ТО, в общем случае, целесообразно, ибо сопровождается получением положительного эффекта. Его размер определяется уровнем квалификации и общей культуры разработчиков программы обновления, исполнителей работ по программе и эксплуатационников, использующих обновленный ТО.

В осуществлении рассматриваемой программы заинтересованы предприятия - владельцев устаревшего оборудования. Руководящие органы всех уровней должны инициировать и поддерживать работы по обновлению изношенного оборудования. Предприятия, активно ведущие работу по повышению эффективности производства, следует стимулировать и поощрять.

Для повышения результативности работ по рассматриваемой проблематике целесообразно расширить исследования в сфере технической геронтологии. Также необходимо в области технической диагностики развивать технологию и средства, доступного в производственных условиях оперативного контроля над всеми показателями 


\section{$\underline{1}$ ПИТАННЯ ТЕОРІЇ, МЕТОДИ ТА АЛГОРИТМИ ЕФЕКТИВНОГО АВТОМАТИЧНОГО} УПРАВЛІННЯ ОБ’ЄКТАМИ ХІМІКО-ТЕХНОЛОГІЧНОГО ТИПУ

уровня $\mathrm{E}_{\mathrm{T}}$ ТО. Необходимо расширить доступность оперативного применения на производстве современных методов и средств неразрушающего контроля свойств конструкционных материалов, состояния элементов конструкции, оценки влияния на них усталостных явлений, воздействия вибрации, жесткого излучения, шумов режимных параметров ТО и др.

Программа обновления действующих ТО может быть охарактеризована рядом параметров. В их числе наиболее важны два параметра: системность, отражающая номенклатуру проводимых на объекте работ, и масштабность, отражающая широту охвата работами парка ТО.

Как известно, при увеличении системности и масштабности работ удельная результативность ресурсовложений повышается.

В механизме программы уровень системности и уровень масштабности работ взаимосвязаны синергетически. Вследствие этого, при расширении системности и масштабности проводимых по программе работ, удельная результативность ресурсовложений будет возрастать. Это важное свойство программы показывает целесообразность расширения сферы ее применения в разных отраслях производства и целесообразность расширения номенклатуры работ на каждом действующем ТО.

Выводы

1. Значительная часть действующего оборудования отработала ресурс работоспособности, поэтому функционирует с невысоким уровнем $\mathrm{E}_{\mathrm{T}}$;

2. Проблема использования резерва в повышении эффективности изношенных ТО становится все более актуальной;

3. Подобный резерв значителен, а его использование в большинстве случаев возможно и техникоэкономически целесообразно;

4. Для исследования возможности и целесообразности модернизации действующего ТО, необходимо располагать сведениями о текущем уровне его $\mathrm{E}_{л}, \mathrm{E}_{\mathrm{H}}$ и $\mathrm{E}_{\text {общ, }}$ а также закономерностью влияния износа на $\mathrm{E}_{\mathrm{T}}$ объекта;

5. Рассмотренный алгоритм повышения эффективности действующих ТО может быть реализован на всех трех этапах формирования $\mathrm{E}_{\mathrm{T}} \mathrm{TO}$;

6. Программа повышения эффективности действующих ТО по потенциальной ценности ожидаемых результатов достойна концептуального статуса;

7. Реализация программы явится одним из продуктивных элементов стратегии обновления основных фондов в отечественном производстве в период перехода к инновационному сценарию его развития.

Литература

1. Воінова С.О. Можливості управління ефективністю технічних об’єктів/ Труды 15-й Междунар. н.-т. конф. «Физические и компьютерные технологии», Харьков, 2-3 декабря 2009г.- Харьков: ХНПК «ФЭД», 2009.C. $393-395$;

2. Воинова С.А. Особенности формирования ресурса технических объектов/ Енергетика та електрифікація, №10, 2012.- С. 40 - 43;

3. Воинова С.А. Техническая геронтология. Потенциал влияния на эффективность функционирования технических объектов/ Матер. за VI Международна научна практична конференция «Найновите постижения на европейската наука-2010», 17 - 25 юни, 2010г. Том 20 «Технологии. Физическа култура и спорт».- София: «Бял ГРАД-БГ» ООД, 2010.- С. 79 - 84;

4. Воинова С.А. Обновление как инструмент развития производства/ Известия вузов и энергетических объединений СНГ, № 2, 2013.- С. 69 - 74.

\section{References}

1. Voinova s.o. mozhlivosti upravlinnya efektivnistyu texnichnix ob'ektiv/ trudy 15-j mezhdunar. n.-t. konf. «fizicheskie i kompyuternye texnologii», xarkov, 2-3 dekabrya 2009g.- xarkov: xnpk «fed», 2009.- s. 393 - 395 ;

2. Voinova s.a. osobennosti formirovaniya resursa texnicheskix obektov/ energetika ta elektrifikaciya, №10, 2012.- s. $40-43$;

3. Voinova s.a. texnicheskaya gerontologiya. potencial vliyaniya na effektivnost funkcionirovaniya texnicheskix obektov/ mater. za vi mezhdunarodna nauchna praktichna konferenciya «najnovite postizheniya na evropejskata nauka-2010», 17 - 25 yuni, 2010g. tom 20 «texnologii. fizicheska kultura i sport».- sofiya: «byal grad-bg» ood, 2010.- s. 79 - 84;

4. Voinova s.a. obnovlenie kak instrument razvitiya proizvodstva/ izvestiya vuzov i energeticheskix obedinenij sng, № 2, 2013.- s. 69 - 74 . 